\title{
Surgical Site Infection Prevention Bundle in Cardiac Surgery
}

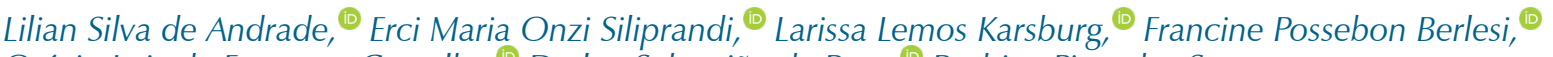 \\ Otávio Luiz da Fontoura Carvalho, ${ }^{[0}$ Darlan Sebastião da Rosa, ${ }^{(0)}$ Rodrigo Pires dos Santos \\ Instituto de Cardiologia do Rio Grande do Sul - Fundação Universitária de Cardiologia (IC/FUC), Porto Alegre, RS - Brazil
}

\begin{abstract}
Background: Surgical site infections (SSI) are among the most prevalent infections in healthcare institutions, attributing a risk of death which varies from $33 \%$ to $77 \%$ and a 2 - to 11 -fold increase in risk of death. Patients submitted to cardiac surgery are more susceptible to SSI, accounting for $3.5 \%$ to $21 \%$ of SSI. The mortality rate attributable to these causes is as high as $25 \%$. Prevention of SSI in cardiac surgery is based on a bundle of preventive measures, which focus on modifiable risks.
\end{abstract}

Objective: The objective of this study was to identify SSI risk factors in clean cardiac surgery.

Methods: A retrospective cohort study analyzed 1,846 medical records from patients who underwent clean cardiac surgery. Fisher's exact test was used for bivariate comparison, and Poisson regression was used for independent analysis of SSI risk, considering a significance level of $p<0.05$.

Results: The results of the study comprised a multivariate analysis. The variables that were associated with the diagnosis of SSI were: surgical risk index (OR: 2.575; CI: 1.224-5.416), obesity (OR: 2.068; CI: 1.457-2.936), diabetes mellitus (OR: 1,678; CI: 1.168-2.409), and blood glucose level (OR: 1.004; Cl: 1.001-1.007).

Conclusions: This study evidenced that complete adherence to the bundle was not associated with a reduction in the risk of surgical infections. Diabetes mellitus, obesity, and surgical risk index assessment were, however, identified to increase association and consequently risk of SSI in cardiac surgery. (Arq Bras Cardiol. 2019; 112(6):769-774)

Keywords: Cardiac Surgical Procedures; Adult; Risk Factors/prevention and control; Patient Care Bundles; Anti-Infective Agents; Surgical Wound Infection; Cross Infection.

\section{Introduction}

Healthcare associated infections ( $\mathrm{HAl}$ ) are defined as any infection that occurs in a patient during the process of care in a health facility within 48 to 72 hours of initial contact with the healthcare system. Infection rates are higher in developing countries and in intensive care units. ${ }^{1,2}$

Surgical site infections (SSI) are among the most prevalent in health institutions. In the United States, in the year 2011, SSI affected an average of 157,500 patients. The risk of death attributable to this type of infection is high, varying from $33 \%$ to $77 \%$, and SSI are associated with a 2 - to 11 -fold increase in risk of death. ${ }^{3}$

Patients who undergo cardiac surgery are particularly susceptible to hospital infections and SSI, which result in further interventions and additional costs for the health institution. SSI rates can vary from $3.5 \%$ to $21 \%$, and the mortality rate due to these causes can reach $25 \%$. ${ }^{4,5}$

Various risk factors are associated with SSI in cardiac surgery, including: age, nutritional status, diabetes mellitus, tobacco use, obesity, coexistence of infections in other sites, length of

Mailing Address: Lilian de Andrade •

Avenida Princesa Isabel, 395, setor SCIH. Postal Code 90040-371, Santana,

Porto Alegre, RS - Brazil

E-mail: lilianandrade16@gmail.com

Manuscript received June 01, 2018, revised manuscript September 05, 2018, accepted November 01, 2018

DOI: $10.5935 / a b c .20190070$ preoperative stay, skin preparation, mechanical ventilation, failure to comply with aseptic techniques, inadequate hand hygiene, distractions in the operating area, number of times doors are opened, and other environment-related factors. ${ }^{6}$

"Bundles" of preventive measures applied to surgical procedures have been effective in reducing infection rates. These measures include: delivering antibiotic prophylaxis within 1 hour before incision, discontinuing antibiotic use within 48 hours after cardiac surgery, performing hair removal during the immediate preoperative period, maintaining intraoperative normothermia of $35.5^{\circ} \mathrm{C}$ or more, and blood glucose control during the immediate postoperative period, extended for 48 hours after the procedure. ${ }^{7}$

The objective of this study was to identify risk factors for SSI in major clean cardiac surgery procedures in a cardiology referral center.

\section{Methods}

This is a retrospective cohort study, conducted in the Instituto de Cardiologia (Cardiology Institute), a hospital with 250 beds for cardiology patients in the South of Brazil. The study evaluated patients who underwent major surgical procedures with and without the use of extracorporeal circulation during the period from January 2013 to December 2014. The study included all major surgeries in adults (age 18 or over). The following were excluded: pediatric patients, patients with incomplete medical records data, patients who died during immediate pre-, intra-, or postoperative, and patients who were hospitalized for less than 48 hours. 
The epidemiological diagnosis of infections followed the North American Center for Disease Control and Prevention (CDC) criteria. Infections were classified as superficial, deep, or organ/space. The institution has used a protocol for the prevention of SSI since 2003.

The bundle of preventive measures at the institution includes the following 6 items to be executed throughout the pre- and postoperative periods: preoperative bath with $2 \%$ chlorhexidine, 24 hours before the procedure; hair removal with electric clippers, within 2 hours prior to the start of surgery; maintenance of normothermia, at least $36^{\circ} \mathrm{C}$, during the immediate postoperative period; controlling blood glucose below $200 \mathrm{mg} / \mathrm{dl}$, measured at 6:00 am on the first postoperative day; infusion of surgical antibiotic prophylaxis with anesthetic induction within 60 minutes prior to incision; and additional doses if the procedure lasts more than 4 hours, with a maximum use time of 24-48 hours.

The risks scores considered were the ASA classification developed by the American Society of Anesthesiologists and the National Nosocomial Infection Surveillance (NNIS) risk index. The NNIS risk index ranges from 0 to 3, taking the following items into account: contamination potential, procedure duration, and ASA classification. Each item is worth either 0 or 1 points in the score. ${ }^{24}$

Data collection made use of the Brazilian Hospital Infection Control Service $(\mathrm{SCIH})$ information system. Additionally, patients' medical records were reviewed using the Brazilian Medical and Statistical Archive Service (SAME). The medical records were reviewed in the second semester of 2015.

\section{Statistical analyses}

Sample size calculation considered the rates of infection between 2003 and 2012. Considering a number of 900 procedures per year in the hospital and an average SSI rate of 3.23\% for the period between 2003 and 2012 and that the complete application of the prevention bundle reduces the infection rate by $60 \%{ }^{8}$ a sample of 1.846 medical records was calculated, with an alpha error of $5 \%$ and a beta error of $20 \%$.

Fisher's exact test was used for bivariate comparison. Poisson regression was used for multivariate analysis which included variables with $p<0.20$ from the bivariate analysis. ${ }^{9}$ A significance level of $p<0.05$ was considered.

The data collected were codified and inserted into a table using the program Microsoft Office Excel 2007, thus creating a databank. Complementary analyses were carried out using the program SPSS, version 18.0.

This study was approved by the research ethics committee of the Instituto de Cardiologia of the Fundação Universitária de Cardiologia, on September 17, 2014, under certificate number 4997/14, being accredited by the Brazilian National Commission of Ethics in Research (CONEP), with the Term of Confidentiality for Data Use attached.

\section{Results}

One thousand, eight hundred, and forty-six medical records of patients who underwent major surgical procedures were analyzed, 138 of which were excluded from the study for the following reasons: 23 pediatric patients, 85 deaths or hospitalizations lasting less than 48 hours, and 30 records with incomplete data which did not meet study inclusion criteria. The period studied, thus, included a total of 1,708 major cardiac surgery procedures in 1,708 patients.

One hundred and forty-two (8.3\%) procedures developed SSI, of which $48.0 \%(n=69)$ were thoracic site infections (13.3\% superficial incisional; $24.5 \%$ deep incisional; $11.2 \%$ organ/space); $40.6 \%(n=58)$ were saphenous vein infections; $7.7 \%(n=9)$ were thoracic site and saphenous vein infections; and $3.0 \%(n=4)$ were endocarditis. In heart transplant procedures, 1 in 4 became infected.

The demographic data of patients with and without the presence of SSI are shown in Table 1.

The following variables correlated with infection in bivariate analysis: arterial hypertension $(p=0.01)$, diabetes mellitus $(p=0.001)$, dyslipidemia $(p=0.05)$, obesity $(p=0.001)$, blood glucose level $\geq 200 \mathrm{mg} / \mathrm{dl}$ ( $p=0.03$ ), public or private hospitalization $(p=0.008)$, surgical risk index $(p=0.001)$. The following variables were associated with the diagnosis of SSI in multivariate analysis: surgical risk index, obesity, diabetes mellitus, and blood glucose level (Table 2).

\section{Discussion}

The SSI rate in our study was $8.3 \%$. In developed countries, the SSI rate varies from $1.2 \%$ to $5.2 \%$, whereas, in developing countries, it may be as high as $11.8 \%$. Our rate was, therefore, higher than the general SSI rate in developed countries $(1.2-5.2 \%)$, but lower than the rate in developing countries $(11.8 \%){ }^{3}$

SSI rates following cardiac surgery in developing countries may vary from $3.5 \%$ to $21.0 \% .^{4,5}$

Diabetes mellitus, blood glucose level, obesity, and surgical risk index are factors associated with SSI, in accordance with the latest World Health Organization report (WHO, 2016), which underlines these factors in relation to risks that affect HAI. ${ }^{3}$

SSI risk factors are complex, and their prevention requires the integration of a range of measures, before, during, and after surgery. Prevention is the principal focus of the Institute for Healthcare Improvement (IHI) and the Surgical Care Improvement Project (SCIP) in the USA, both of which recommend a group of preventive measures to be taken. ${ }^{10}$ These measures, called a "bundle," are carried out together to obtain better results than would be obtained by individual application. SSI prevention bundles in cardiac surgery involve the use of prophylactic antibiotics during the immediate pre-and postoperative period (up to 48 hours following incision); blood glucose level control during the first and second postoperative period; temperature and oxygenation control; decolonization of patients with intra-nasal mupirocin, and preoperative chlorhexidine bath. ${ }^{3,7,10}$

In our study, adherence to surgical prophylaxis protocol was not associated with a reduction in SSI rates. Studies in surgical procedures indicate that antimicrobial use within 60 minutes before the procedure has been associated with reduced infection rate..$^{3,11,12}$ 


\section{Original Article}

Table 1 - Sociodemographic data associated with SSI

\begin{tabular}{lccc}
\hline & SSI (N/\%) & No infection (N/\%) & Total (N) \\
\hline Male sex & $92(64.8 \%)$ & $1,057(67.9 \%)$ & 1,149 \\
Type of procedure & & & \\
- Myocardial revascularization & $85(8.4 \%)$ & $923(91.6 \%)$ & 1,008 \\
- Valve replacement & $49(34.5 \%)$ & $545(35.0 \%)$ & 594 \\
- Aortic dissection & $8(5.6 \%)$ & $88(5.7 \%)$ & 96 \\
Hypertension & $133(94.3 \%)$ & $1,345(87.2 \%)$ & 1,478 \\
Tobacco use & $63(44.7 \%)$ & $620(40.2 \%)$ & 683 \\
Diabetes mellitus & $75(53.2 \%)$ & $484(31.4 \%)$ & 559 \\
Dyslipidemia & $58(41.1 \%)$ & $504(32.7 \%)$ & 562 \\
Obesity & $38(27.1 \%)$ & $169(11.0 \%)$ & 207 \\
COPD & $7(5.0 \%)$ & $70(4.5 \%)$ & 77 \\
Renal insufficiency & $9(6.3 \%)$ & $84(5.4 \%)$ & 93 \\
Public healthcare & $112(78.9 \%)$ & $1,061(68.2 \%)$ & 1,173 \\
ASA class III & $111(78.2 \%)$ & $1,282(82.4 \%)$ & 1,393 \\
Adequate use of antibiotic bundle & $33(23.2 \%)$ & $332(21.4 \%)$ & 365 \\
Complete adherence to total bundle & $6(4.3 \%)$ & $61(3.9 \%)$ & 67 \\
Death & $11(7.7 \%)$ & $165(10.6 \%)$ & 176 \\
\hline
\end{tabular}

SSI: surgical site infection; COPD: chronic obstructive pulmonary disease; ASA: American Society of Anesthesiologists.

Table 2 - Multivariate Analysis. Poisson Regression

\begin{tabular}{lccc}
\hline Risk Factor & Odds Ratio (OR) & Confidence Interval (IC) & $p$ \\
\hline Surgical risk index & 2.575 & $1.224-5416$ & 0.013 \\
Public vs. private healthcare & 1.473 & $0.974-2.229$ & 0.067 \\
Systemic arterial hypertension & 1.770 & $0.877-3.573$ & 0.111 \\
Diabetes mellitus & 1.678 & $1.168-2.409$ & 0.005 \\
Dyslipidemia & 1.083 & $0.777-1.510$ & 0.637 \\
Obesity & 2.068 & $1.457-2.936$ & $<0.001$ \\
Adequate glycemia (<200 mg/dl) & 1.077 & $0.724-1.601$ & 0.715 \\
Blood glucose level & 1.004 & $1.001-1.007$ & 0.007 \\
\hline
\end{tabular}

In our study, $96 \%$ of patients used either a first or secondgeneration cephalosporin. Meta-analysis evidenced that the use of cefuroxime as a cardiac surgery prophylaxis demonstrated better protection against respiratory infections in the immediate preoperative period. Although our study did not evaluate this type of outcome, there was no difference in the comparison between cefazolin (institutional protocol effective before May 2014) and cefuroxime (institution recommendation as of June 2014) for SSI (data not shown).

The prevention bundle used at our research institution included six preventive measures. Complete adherence to the bundle was not associated with reduced risk of surgical infections. Regarding the CDC's bundle of measures, it establishes the following preventive measures with respect to the perioperative period: surgical antibiotic prophylaxis during the pre-, intra-, and postoperative periods; performing hair removal when necessary, without the use of razors; blood glucose level control during the pre- and postoperative periods; normothermia throughout the perioperative period; optimization of tissue oxygenation; skin preparation with alcoholic solutions; and finally the use of the Surgical Safety Checklist. ${ }^{3}$

Blood glucose level alone was associated with a reduced surgical infection risk. Nonetheless, dichotomized levels below $200 \mathrm{mg} / \mathrm{dl}$ were not associated with reduced infection rates. Various studies have associated blood glucose level with risk of infection in cardiac surgery. There is recent evidence that strict blood glucose control (levels below $180 \mathrm{mg} / \mathrm{dl}$ ) can 
reduce SSI rates in patients who undergo surgical procedures. ${ }^{3}$ Furthermore, confirming the findings of our study, diagnosis of diabetes mellitus, regardless of blood glucose level, increases the risk of SSI in cardiac surgery. The SCIP, which was founded in 2003 as a national-level partnership of organizations committed to improving surgical care safety by reducing postoperative complications, developed a core measure to maintain blood glucose level at $\leq 180 \mathrm{mg} / \mathrm{dL}$ during the perioperative and postoperative periods, based on evidence that this reduces SSI in cardiac surgery. ${ }^{10,13,14}$ The Society for Healthcare Epidemiology of America (SHEA) and the Infectious Diseases Society of America (IDSA) also recommend blood glucose level goal of $\leq 180 \mathrm{mg} / \mathrm{dL}$ during the immediate postoperative period. According to a study conducted by the Society of Thoracic Surgeons, it has been demonstrated that maintaining blood glucose levels between $150 \mathrm{mg} / \mathrm{dL}$ and $180 \mathrm{mg} / \mathrm{dL}$ reduces the risk of SSI in cardiac surgery. ${ }^{7,15} \mathrm{~A}$ randomized controlled study of 5,510 diabetic cardiac surgery patients, with an intravenous insulin protocol used to maintain blood glucose at $\leq 150 \mathrm{mg} / \mathrm{dL}$, demonstrated that the use of this protocol is safe and that it led to a $77 \%$ reduction in $\mathrm{SSI}{ }^{16}$

Obesity is a risk factor for SSI in cardiac, colorectal, orthopedic, caesarean section, and general surgery, as the procedure becomes more complex and increases the duration of surgical stay, causing tissue hypoxia and hyperglycemia related to the obese patients' insulin resistance, thus, contributing to the risk of SSI. Obese patients have a higher risk of acquiring infections, especially when they are exposed to surgical procedures and hospitalization in intensive care units. ${ }^{17-19}$ A study conducted in the USA demonstrated that risk of infection was 4.7 times higher in obese surgery patients and 6 times higher in surgery patients with morbid obesity, in comparison with normal-weight surgery patients. ${ }^{19}$ Furthermore, a recent study followed up 33,936 patients after myocardial revascularization surgery, showing that the factors that determined high risk of surgical infection included female sex, obesity, unplanned reoperations, and longer hospital stays. ${ }^{20}$ In our study, the risk of infection related to obesity was 1.8 times higher.

The surgical risk index is a good predictor of risk in surgeries. In one study, the use of NNIS risk index contributed to the stratification of SSI incidence rates in cardiac surgery. In this study, the incidence of mediastinitis was $0 \%$ when the patient's score was $0,1.2 \%$ when it was 1 , and $2.3 \%$ when it was $2 .{ }^{21,22}$ Our study demonstrated that in clean surgeries, the component related to base pathologies, patient's physical state (ASA class), and procedure duration, as measured by NNIS risk index, was associated with a higher risk of infection.

Although obesity and diabetes mellitus are modifiable factors in most cases, these pathologies may signal greater risks. Healthcare professionals may then take greater care with the patient, for example, with strict attention to the surgical technique, dead space reduction, tissue circulation, and postoperative care.
There are some limitations to our study. It is a retrospective study of only one cardiology center. Although we assessed the patients' physical state and risk factors (ASA class) and procedural risks (length of surgical stay), the final analysis did not include some risk factors, such as patient skin antisepsis (preoperative); the surgical team's abilities and operating room assistants' behavior (intraoperative); surgical sterilization practices; the use of invasive procedures during the postoperative period, such as catheters, probes, or mechanical ventilation; and other risk factors related to infection.

\section{Conclusion}

Obesity, diabetes, and blood glucose level were independent factors associated with SSI in patients who underwent major cardiac surgery procedures. Surgical risk index was a good predictor score for SSI in cardiac surgery. Surgical antibiotic prophylaxis and adherence to the SSI prevention bundle for cardiac surgery were not associated with a decrease in SSI risk.

\section{Author contributions}

Conception and design of the research and Critical revision of the manuscript for intellectual content: Andrade L, Siliprandi EMO, Pires R; Acquisition of data: Andrade L, Siliprandi EMO, Karsburg LL, Berlesi FP, Carvalho OLF, Rosa DS, Pires R; nalysis and interpretation of the data and Obtaining financing: Andrade $L$, Pires R; Statistical analysis: Andrade L, Karsburg LL, Berlesi FP, Pires R; Writing of the manuscript: Andrade L, Siliprandi EMO, Carvalho OLF, Rosa DS, Pires R.

\section{Potential Conflict of Interest}

No potential conflict of interest relevant to this article was reported.

\section{Sources of Funding}

There were no external funding sources for this study.

\section{Study Association}

This article is part of the thesis of master submitted by Lilian Silva de Andrade, from Instituto de Cardiologia do Rio Grande do Sul.

\section{Ethics approval and consent to participate}

This study was approved by the Ethics Committee of the Instituto de Cardiologia - Fundação Universitária de Cardiologia - Unidade de Pesquisa under the protocol number 4997/14. All the procedures in this study were in accordance with the 1975 Helsinki Declaration, updated in 2013. Informed consent was obtained from all participants included in the study. 


\section{Original Article}

\section{Referências}

1. Allegranzi B. Report on the load associated infections endemic health care worldwide. Genebra: WHO ;2011.

2. Horan TC, Andrus M, Dudeck MA. CDC surveillance definition / NHSN infections associated with health care and criteria for specific types of infections in the intensive care setting. Am J Infect Control.2008;36(5):309-32

3. World Health Organization. (WHO) Global Guidelines for the Prevention of Surgical Site Infection . [Cited in 2018 Feb 23] Available from: www.who.int

4. Surveillance of surgical site infections in Europe 2010-2011. Stockholm: European Centrefor Disease Prevention and Control; 2013[Cited in 2018Aug 21] Available from: (https://ecdc.europa.eu/sites/portal/files/media/en/publications/ Publications/120215_TED_SSI_protocol.pdf(-in-europe-2010-2011.

5. Le Guillou V, Tavolacci MP, Baste JM, Hubscher C, Bedoit E, Bessou JP, et al. Surgical site infection after central venous catheter related infection in cardiac surgery. Analysis of a cohort of 7557 patients. J Hosp Infect. $2011 ; 79(3): 236-41$.

6. National Institute for Health and Care Excellence (NICE). Clinical Guideline. Surgical site infection prevention and treatment of surgical site infection.[Cited in 2017 Jan 10]. Available from: https://www.nice.org.uk/ guidance/og74.

7. Anderson DJ, Podgorny K, Berrios-Torres SI, Bratzler DW, Dellinger EP, Greene L, et al. Strategies to prevent surgical site infections in acute care hospitals: 2014 update. Infect Control Hosp Epidemiol. 2014;35(6):605-27.

8. Anderson DJ, Podgorny K, Berríos-Torres SI, Bratzler DO, Dellinger EP, Greene L, et al. Strategies to Prevent Surgical Site Infections in Acute Care Hospitals: 2014. Infect Control Hosp Epidemiol.2014;35(6):605-27.

9. McCullagh P, Nelder J. Generalized linear models. $2^{\text {nd }}$ ed Flórida, EUA: Chapman \& Hall; 1989.

10. The Joint Commission. Surgical Projeto de Melhoria Care. [Internet] [Cited in 2014 Mar 30] Available from: http://www.jointcommission.org/ surgical_care_improvement_project/).

11. Organização Mundial Da Saúde. (OMS) Segundo desafio global para a segurança do paciente: Cirurgias seguras salvam vidas (orientações para cirurgia segura da OMS) . Brasilia; 2009.
12. AJ Mangram, TC Horan, ML Pearson, Silver LC, Janvis WR. Guidance for the prevention of surgical site infection, 1999. Hospital Advisory Committee infection control practices. Infect Control Hosp Epidemiol. $1999 ; 20(4): 250-78$.

13. Latham R, Lancaster AD, Covington JF, Pirolo JS, Thomas CS Jr. et al. The association of diabetes and glucose control with surgical-site infections among cardiothoracic surgery patients. Infect Control Hosp Epidemiol. 2001;22(10):607-12.

14. Centers for Disease Control and Prevention. Atlanta, GA;2014. [Cited in 2018 April 10] Available from: http://www.cdc.gov/diabetes/pubs/ statsreport14/national-diabetes-report-web.pdf

15. L Lazar L, McDonnell M, Chipkin S,. The Society of Thoracic Surgeons practice guideline series: glucose management in blood during cardiac surgery in adult patients. Ann Thorac Surg.2009;87(2):663-9.

16. Furnary AP, WuY. The clinical effects of hyperglycemia on population cardiac surgery: the Diabetic Project Portland. Endocr Pract. 2006;12(Suppl 3):22-6.

17. Newell MA, Bard MR, Goettler CE, Toschlog EA, Schenarts PJ, Sagraves SG, et al. Body man index and outcomes in critically injured blunt trauma patients. J Am Coll Surg. 2007;(5):1056-61.

18. Bistrian, B. Answer systemic inflammation. Revi Nutri. 2007;65(supl):170-2.

19. Pablo E. Serrano PE, Khuder AS, Sadik A. Khuder. John J. Fath obesity as a risk factor for nosocomial infections in trauma patients. Abstract presented at American College of Surgeons. J Am Coll Surg.2010;211(1):61-7.

20. Hannan EL, Zhong Y, Lahey SJ, Culliford AT, Gold JP, Smith CR, et al. 30-day readmissions after coronary artery bypass graft surgery in New York State. JACC Cardiovasc Interv. 2011;4(5):569-76.

21. Roy MC, Herwaldt LA, Embrey R, Kuhns K, Wenzel RP, Perl TM. Does the Centers for Disease Control and Prevention's NNIS System risk index stratify patients undergoing cardiothoracic operations by their risk of surgical-site infection? Infect Control Hosp Epidemiol. 2000;21(3):186-90.

22. Lepelletier D, Perron S, Bizouarn P, Caillon J, Drugeon H, Michaud JL, et al. Surgical-site infection after cardiac surgery: incidence, microbiology, and risk factors. Infect Control Hosp Epidemiol 2005;26(5):466-72. 
